\title{
EchoGéo
}

\section{Les réfugiés dans le monde entre protection et illégalité}

\section{Olivier Clochard}

\section{Q OpenEdition}

\section{Journals}

Édition électronique

URL : https://journals.openedition.org/echogeo/1696

DOI : 10.4000/echogeo.1696

ISSN : 1963-1197

\section{Éditeur}

Pôle de recherche pour l'organisation et la diffusion de l'information géographique (CNRS UMR 8586)

Référence électronique

Olivier Clochard, «Les réfugiés dans le monde entre protection et illégalité », EchoGéo [En ligne], 2 | 2007, mis en ligne le 22 février 2008, consulté le 31 juillet 2021. URL : http://journals.openedition.org/ echogeo/1696; DOI : https://doi.org/10.4000/echogeo.1696

Ce document a été généré automatiquement le 31 juillet 2021.

EchoGéo est mis à disposition selon les termes de la licence Creative Commons Attribution - Pas d'Utilisation Commerciale - Pas de Modification 4.0 International (CC BY-NC-ND) 


\title{
Les réfugiés dans le monde entre protection et illégalité
}

\author{
Olivier Clochard
}

1 Selon les chiffres du Bureau international du travail et des Nations unies (2006), sur une population de 6,5 milliards de femmes et d'hommes dans le monde en 2005 , le nombre de migrants internationaux est estimé à près de 200 millions (soit près de $3 \%$ des habitants de la planète). Au cours des quarante dernières années, les effectifs des migrants internationaux se caractérisent par une progression continue ${ }^{1}$; sur un plan démographique, il convient toutefois de relativiser cette augmentation au regard de l'accroissement du nombre d'habitants dans le monde ${ }^{2}$. L'attrait exercé par des emplois mieux rémunérés dans les pays développés et en voie de développement (Emirats Arabes Unis, Inde...) est l'un des principaux moteurs des migrations internationales (cf. carte 1). Selon les Nations unies (2006, p. 13), sur 191 millions de migrants internationaux, 11 millions vivent dans les pays développés et 75 millions dans des pays en voie de développement. Ces chiffres n'englobent pas les demandeurs d'asile, les réfugiés et les personnes déplacées dans le monde. Les recherches sur les demandeurs d'asile et les réfugiés sont étayées par d'importants travaux (Agier M./2002 ; Lassailly Jacob V. et al./1999 et 2001 ; Legoux L. / 2002, 2004 et 2006 ; Le Pors A./2005 ; Rodier C./ 2005 et 2006 ; Valluy J./ 2004 et 2006...) ; une littérature qui parfois, doit beaucoup aux témoignages et aux analyses des ONG ou des associations de défense des réfugiés comme en France (Anafé ${ }^{3}$, Coordination Française pour le Droit d'Asile ${ }^{4}$, la Cimade ${ }^{5}$, le Gisti ${ }^{6}$...). En 2004, parmi les pays développés, la France a accueilli le plus grand nombre de demandeurs d'asile (soit 58550 demandes contre 30910 en 1999) ; alors que les effectifs des États tels la Grande-Bretagne et l'Allemagne, diminuaient par rapport aux années précédentes ${ }^{7}$. Suite à plusieurs réformes législatives, le nombre annuel de demandeurs d'asile en France va connaître à son tour une baisse importante conduisant à 42000 enregistrements en 2005 et moins de 27000 en 2006. Ce processus va amener le ministre de l'Intérieur français à dire à l'ensemble des préfets en juillet $2006^{8}$, que "c'est un véritable succès qui couronne nos efforts depuis quatre ans dans ce domaine et nous rapproche enfin de nos partenaires européens, en particulier le Royaume-Uni et l'Allemagne ». Or de tels propos peuvent être qualifiés d'indécents si nous replaçons ces différents 
chiffres dans un cadre regroupant l'ensemble des continents. Quelle est en effet la répartition démographique des réfugiés et des personnes déplacées dans le monde ? Les États occidentaux sont-ils réellement confrontés à de véritables flux de demandeurs d'asile comme le laissent entendre implicitement de nombreux discours officiels ? Pour répondre à ces interrogations souvent évoquées, nous commencerons tout d'abord par une mise en perspective du poids démographique et de la répartition spatiale des réfugiés dans le monde. Puis nous nous intéresserons à l'évolution du droit d'asile aux frontières des Etats européens.

1. La place des demandeurs d'asile, des réfugiés et des personnes déplacées dans les migrations internationales

2 Les migrations de crise sont un autre aspect important des mouvements de population dans le monde ; c'est "l'un des moteurs [souvent] terribles de la dynamique des migrations humaines» (Simon G., 1995, p. 107). En 2004, le nombre de demandeurs d'asile, de réfugiés et de personnes déplacées dans le monde avoisine les 50 millions de personnes. À la différence de la répartition spatiale des migrants internationaux, les populations réfugiées représentées sur la carte 2 , se situent majoritairement dans les pays en voie de développement comme les États du Proche-Orient (Jordanie, Liban, Palestine), l'Iran, le Pakistan ou la Tanzanie. Cette différence entre le Nord et le Sud est encore plus nette pour les populations déplacées à l'intérieur des frontières de leur pays (et représentée sur la carte 3) ; au Soudan, dans la région des Grands Lacs (Ouganda, RDC), en Colombie et plus récemment au Liban et en Irak, les personnes déplacées sont de l'ordre de plusieurs millions. En octobre 2006, le Haut commissariat des Nations unies pour les réfugiés (HCR) souligne que 754000 Irakiens ont été déplacés à cause de la violence en Irak depuis l'invasion américaine en mars 2003 ; et en Syrie, le nombre de réfugiés irakiens est estimé à près de 450000 personnes $^{9}$. Entre 1945 et 1990, les déplacements des réfugiés trouvaient principalement leur origine dans les nombreuses crises résultant de confrontations idéologiques, politiques et militaires entre l'Est et l'Ouest. Après avoir été multiplié par trois entre 1948 et 1991, le nombre de conflits armés sur la planète a diminué depuis l'arrêt de la guerre froide en novembre 1989 et l'effondrement de l'URSS en décembre 1991. Toutefois les guerres civiles, aujourd'hui plus nombreuses que les affrontements entre États, les crises humanitaires, les nouvelles formes de conflits (comme les guerres menées par les États-Unis d'Amérique pour lutter contre le terrorisme international) entraînent des exodes et des déplacements contraints de populations de plus en plus importants : moins 3 millions de personnes relevaient de la compétence du HCR en 1976; près de 12 millions en 1985 et plus de 19 millions en 1997. À la fin de l'année 2004, le nombre de réfugiés, de demandeurs d'asile et autres personnes relevant de la compétence du HCR était de 19,2 millions (20,8 millions à la fin de l'année 2005 dont près de $50 \%$ de réfugiés); ce chiffre ne tient pas compte d'un grand nombre de personnes déplacées à l'intérieur de leur pays, des quatre millions de Palestiniens qui sont sous la protection de l'UNRWA, ni des exilés vivant de façon clandestine en dehors des frontières de leur État.

3 Les exilés victimes de déplacements liés aux guerres et à la violence, sont généralement désignés comme des " réfugiés "; mais cette dénomination estompe d'autres catégories traditionnellement utilisées par le HCR comme " apatrides ", "personnes déplacées " ou «demandeurs d'asile». Selon le graphique 1, les personnes déplacées (c'est-à-dire celles qui s'installent dans une autre région de leur État) composent le groupe le plus important des exilés dans le monde en 2004:5,4 millions d'entre eux sont pris en 
charge par le Haut commissariat aux Réfugiés mais 20 à 30 millions de déplacés ne bénéficient pas (ou peu) de l'assistance des ONG et/ou de la protection des Nationsunies. Le second groupe concerne les réfugiés et les apatrides ayant quitté leur État d'origine pour se présenter aux frontières d'un autre État où ils ont demandé l'asile. Rappelons que les deux notions asile et réfugié sont à la fois distinctes et liées. Distinctes parce que "l'asile est un espace, le réfugié est une personne" (Alland D., 1997, p. 15). Une personne peut être réfugiée sans avoir le droit d'asile sur un autre État; les États signataires de la convention de Genève de 1951 ne sont pas obligés d'accorder le droit au séjour aux réfugiés se présentant sur leur territoire. La question de l'asile reste une prérogative de l'État. L'accueil des demandeurs d'asile (dont l'effectif en 2004 avoisine les 840000 personnes) est donc subordonné à une autorisation provisoire de séjour des pays d'accueil avant que ces derniers voient éventuellement leur droit d'asile consacré par la reconnaissance de qualité de réfugié ou par le bénéfice d'un autre statut protecteur (Le Pors A., 2005). En 2004 et 2005, la France est l'État européen qui accueille le plus grand nombre de demandeurs d'asile (avec respectivement 58000 et 50000 demandeurs d'asile), une situation qui semble gêner le Premier Ministre et le ministre de l'Intérieur français, alors que l'accueil des réfugiés en France (et plus largement en Europe) occupe une modeste place. Le 18 avril 2007, lors d'une conférence relative à la situation en Irak et organisé par le HCR à Genève, les pays industrialisés n'ont pas proposé d'accueillir davantage de réfugiés irakiens; "la Commission européenne a indiqué être favorable à un accueil de réfugiés en Europe mais a souligné ne pas avoir d'instrument juridique pour obliger les États à le faire $»^{10}$. Sur 3 millions d'exilés irakiens dans le monde, leur nombre est estimé à 22220 personnes dans les pays industrialisés à la fin de l'année 2006, soit 0,74\% de l'effectif total ; la majeure partie se situant en Jordanie et en Syrie.

Demandeurs d'asile, réfugiés, personnes déplacées dans leur propre pays, personnes sous la protection des Nations unies... chacun de ses termes renvoie à une situation précise et emboîtée (ou non) statistiquement dans une autre (cf. graphique 1). Les effectifs de ces catégories ne cessent d'évoluer en raison des guerres et des violences dans le monde qui se succèdent. Les organisations internationales comme le HCR, les ONG ou les États peuvent aussi modifier l'importance numérique de ces catégories. En 1991, une zone de sécurité est établie dans le nord de l'Irak sous l'égide des Nations unies afin de limiter l'exode des Kurdes et protéger les personnes déplacées venues s'y réfugier. Lors d'un colloque au début du mois de novembre à Marseille consacré à la rétention et l'immigration dans les pays du Maghreb, Michel Agier ${ }^{11}$ rappelait qu'en 2002, 130000 Afghans vivant au Pakistan depuis plusieurs années et ne bénéficiant pas de la protection des Nations unies, ont été reconnus comme réfugiés par le HCR. Ces nombreux exilés peuvent aussi passer d'une catégorie à une autre notamment lorsqu'ils franchissent les frontières des États. À l'aéroport de Roissy, des réfugiés palestiniens dont la vie est devenue très pénible (au Liban ou à Gaza) et demandant à entrer sur le territoire français au titre de l'asile, sont parfois rejetés dans l'illégalité (exemple 1). À l'été 2006, période durant laquelle se déroule la guerre entre l'Israël et le Liban, de nombreuses demandes d'asile de Libanais ont été rejetées à l'aéroport de Roissy ${ }^{12}$; alors que le gouvernement français avait adressé le 20 juillet aux préfets une circulaire les invitant à "délivrer aux ressortissants libanais présents en France qui ne disposeraient pas d'un droit de séjour [...] une autorisation de séjour d'un mois, renouvelable, jusqu'à ce que la situation se stabilise au Proche Orient ». Lorsque ces déboutés ne sont pas renvoyés dans leur pays, certains d'entre eux viennent augmenter le nombre d'étrangers vivant en 
situation irrégulière que le ministère de l'Intérieur estimait en juin 2006, entre 200000 et 400000 personnes. Le ministère de l'Intérieur voit d'ailleurs ces déboutés dont certains n'ont d'autre solution que de vivre clandestinement, comme un indice de pression migratoire. Au mois de novembre 2006, un Irakien retenu en zone d'attente à l'aéroport de Roissy, a pu finalement déposer sa demande d'asile uniquement après avoir saisi le juge des référés du tribunal administratif de Cergy-Pontoise. Le 27 novembre, le tribunal administratif a en effet suspendu la décision du ministère de l'Intérieur du 10 novembre qui lui refusait l'accès au territoire français au titre de l'asile. Le deuxième exemple indiqué sur le graphique fait notamment référence à des réfugiés soudanais au Liban qui sont dans l'attente d'une réinstallation; ils sont reconnus par le HCR mais ne possèdent pas de permis de résidence. Et en France ou en Grande Bretagne, la plupart des réfugiés du Darfour voient leur demande d'asile refusée par les autorités compétentes alors que les responsables politiques ne cessent de souligner la situation dramatique de cette région centrale d'Afrique (dont le revenu national brut par habitant est l'un des plus faible de la planète). Ainsi dans le contexte actuel où les lois sur l'asile en Europe sont amendées de façon restrictive, l'étranger qui présente une demande tardive peut se voir notifier un refus d'admission au séjour et/ ou être placé dans un centre de rétention administrative (cf. l'encadré ci-dessous).

Un homme de nationalité soudanaise (M. A. B.) originaire de la région du Darfour, a déposé une demande d'asile le 10 décembre 2004 à Niort. L'OFPRA lui a notifié un refus le 18 février 2005. Logeant dans différents centres d'hébergement d'urgence du département des Deux-Sèvres (situés à 60 ou $70 \mathrm{~km}$. de Niort), il n'est pas allé chercher la décision de l'OFPRA au centre de domiciliation RESO (Niort). Il n'a donc pas pu formuler un recours auprès de la CRR, dans la période qui lui était impartie (un mois). Il est alors allé en Espagne; il a vécu à Madrid dans un foyer de demandeurs d'asile et dans diverses structures jusqu'au jour où il a fait l'objet d'une procédure de réadmission vers la France, mis en œuvre par les autorités espagnoles (dans le cadre du règlement de Dublin). Lorsque il est arrivé à l'aéroport Charles de Gaulle à Roissy, l'agent de la police aux frontières après avoir pris différents renseignements sur les documents qu'il avait en sa possession, l'a laissé entrer sur le territoire. Au printemps de l'année 2006, il a souhaité faire une demande de réexamen de sa demande d'asile ; mais n'ayant pas l'original de la décision du 18 février 2005, une demande de duplicata a été envoyée à l'OFPRA.

Le lundi 26 juin au matin, il s'est présenté à la préfecture pour demander un dossier de réexamen de demande d'asile. Lors de l'entretien avec l'agent de la préfecture, il lui a été dit que s'il déposait une demande de réexamen, il serait placé dans le local de rétention administrative du commissariat de Niort. L'agent de la préfecture lui a notamment proposé de réfléchir 24 heures avant de prendre sa décision, en le laissant repartir. M. A. B. a alors répondu qu'il souhaitait que sa demande d'asile soit réexaminée par L'OFPRA. N'ayant pas le temps de traiter immédiatement sa demande, la préfecture lui a demandé de repasser en début d'après-midi. Un dossier de demande de réexamen lui a alors été remis en début d'après-midi ; et l'agent de la préfecture a demandé à M. A. B. de l'accompagner au commissariat de police de Niort situé à proximité de la préfecture des Deux-Sèvres. Placé en rétention administrative, l'OFPRA lui a notifié un nouveau refus le 6 juillet 2006. Il est sorti du centre de rétention administrative de Plaisir le 18 juillet 2006. Il a ensuite déposé le 3 août 2006 pour la première fois un recours contre la décision de l'OFPRA. En septembre 2006, il a été arrêté par la police dans un parc; et il a fait l'objet d'un nouveau placement en rétention administrative (à Niort puis à Vincennes pendant 30 jours) alors que l'instruction de son recours n'était pas terminée.

(Source : enquête de terrain, mai 2005-janvier 2007) 
2. Les demandeurs d'asile aux frontières des États européens

5 Avec les changements socio-économiques et géopolitiques qui se sont opérés depuis le milieu des années 1980, les États européens sont moins enclins à accueillir ces populations. Pour les cinq dernières années, les 25 États membres de l'Union européenne ont accueilli près de 438990 demandeurs d'asile en 2001, 425540 en 2002, 346690 en 2003, 279860 en 2004 et 237840 en 2005 (cf. graphique 2). En cinq ans, les demandes déposées dans les États membres ont diminué de moitié pour atteindre leur niveau le plus bas depuis 1988. Cette diminution est notamment liée aux dispositifs de contrôles qui sont établis aux frontières des États. Les demandeurs d'asile - parce qu'ils ont franchi (ou tentent de franchir) la frontière clandestinement et/ou n'ont pas de document de voyage en règle - sont de plus en plus considérés par les agents de la Police Aux Frontières et/ou les gouvernements européens comme des étrangers usant abusivement de la procédure d'asile pour entrer sur le territoire d'un État. Les expressions "faux demandeurs d'asile", "faux réfugiés", "réfugiés économiques", "demande d'asile manifestement infondée », "clandestins » sont de plus en plus employées dans les médias et chez certains politiciens; elles nient le droit - octroyé aux demandeurs d'asile - d'entrer irrégulièrement sur le territoire d'un État signataire de la Convention de Genève afin d'y solliciter une protection. En avril dernier, le Haut commissariat des Nations unies pour les réfugiés (HCR) a sévèrement critiqué les "restrictions de plus en plus sévères" au droit d'asile imposées par les pays riches. Dans un rapport intitulé «Les réfugiés dans le monde: les déplacements humains du nouveau millénaire » (HCR, 2006), il est constaté que les pays riches entretiennent une confusion croissante entre les notions de «migrants» et "réfugiés». Le HCR précise que les "réfugiés» y sont souvent "décrits [...] comme des migrants illégaux, des terroristes potentiels et des criminels, ou, au mieux, comme des fraudeurs $»^{13}$. Les autorités des États européens considèrent que de nombreux demandeurs d'asile ne correspondent pas aux critères définis dans la Convention de Genève relative au statut de réfugié. En préjugeant ainsi de la situation d'un grand nombre de demandeurs d'asile aux frontières de l'UE, les gouvernements européens communiquent sur la nécessité de mettre en place des dispositifs aux frontières de l'espace européen ou dans les pays voisins afin de reconnaître les étrangers ayant besoin d'une protection de ceux migrant pour des raisons économiques. Cette conception manichéenne de la migration internationale estompe le fait que beaucoup d'individus fuient leur pays pour des facteurs complexes et non pas exclusivement pour des raisons de persécution. Pour le dire autrement, les persécutions auxquelles les exilés doivent faire face sont de toutes natures. En 1997, le délégué du HCR en France - Darioush Bayandor - soulignait déjà que les demandeurs d'asile "sont de plus en plus les produits d'un engrenage où les échecs économiques et la répression politique s'alimentent mutuellement, leur rendant la vie insupportable. La distinction avec l'émigrant économique alors s'estompe et devant l'étendue du phénomène, les pays d'accueil se referment» (1997, p. 4). De nos jours, un ressortissant soudanais peut voir sa demande d'asile rester lettre morte en Europe ; un communiqué du HCR l'a notamment rappelé en décembre 2005.

6 Les dispositifs matériels et législatifs visant à renforcer la lutte contre l'immigration clandestine empêchent un grand nombre d'étrangers de bénéficier de leurs droits humains, et leur imposent le statut de délinquant. Les droits fondamentaux ne sont plus respectés ; sans les dénoncer explicitement, les États européens créent même des lois visant à les contourner. Ainsi le droit d'asile prévu par la Convention de Genève de 
1951 sur les réfugiés, et le droit reconnu à toute personne de «quitter tout pays, y compris le sien, et de revenir dans son pays » consacré par la Déclaration universelle des droits de l'homme, sont particulièrement menacés. Les derniers développements des politiques menées par l'Union européenne et ses États membres soulignent ce processus. Aux frontières orientales et méridionales de l'Union européennes, des coopérations policières et militaires entre Etats européens et pays voisins, sont mises en place afin de dissuader les candidats à l'exil. Les opérations sous l'égide de Frontex (Agence européenne du gestion des frontières extérieurs) permettent aux Etats européens de faire pression chez les pays voisins, conforter les contrôles des demandes d'asile à la frontière, restreindre le nombre d'arrivées dans les pays européens et s'accorder sur une politique commune d'éloignement des étrangers arrivant en situation irrégulière. L'Union européenne a confirmé officiellement sa volonté de mettre en oeuvre ces dispositions, en adoptant, en novembre 2004, le programme de la Haye. Ce plan pluriannuel qui fixe, pour les 5 années à venir les principales orientations de la politique d'asile et d'immigration de l'Union, recommande notamment de disposer d'une « dimension externe de la politique d'asile et d'immigration ». À ce propos, le réseau Migreurop signale que l'expression d' "émigration illégale» commence à se banaliser ${ }^{14}$ "transformant le seul fait de prendre la route en un acte répréhensible. [...] La criminalisation du migrant à la source n'est certes pas nouvelle. Mais elle a été et reste l'apanage des pays s'inspirant du modèle soviétique, où il est naturel de tirer à vue sur l'émigrant, considéré comme un fuyard; divers régimes autoritaires s'en sont inspirés. La Dudh ${ }^{15}$ a pu servir précisément à cela : «illégal » chez lui, l'émigrant devenait un immigrant bienvenu dans le pays d'accueil. Par une ironie habituelle dans l'histoire politique, une même référence à l'illégalité du départ est invoquée maintenant comme un péché contre notre hospitalité. Et les principes juridiques universellement reconnus sont passés à la trappe $»^{16}$. L'inflation des discours sécuritaires qui ont suivi les évènements du 11 septembre 2001, ont entraîné les demandeurs d'asile à être régulièrement inclus, de façon implicite, dans les problématiques de la criminalisation et du terrorisme ${ }^{17}$. Certains responsables politiques et médias, des policiers et des services administratifs chargés d'instruire les demandes des personnes considèrent l'arrivée ou la présence de ces exilés comme une menace à l'ordre public ${ }^{18}$. Qu'ils soient réfugiés, déplacés, clandestins, sinistrés, illégaux ou légaux, «les individus assignés à ces catégories font alors l'objet de stigmates sociaux et polémiques publiques dans les opinions et les milieux politiques aussi bien en France et en Europe que dans le reste du monde » (Agier M., 2005, p. 97).

Conclusion

7 Les distributions spatiales des réfugiés et des personnes déplacées dans le monde soulignent que les Etats occidentaux ne sont pas les pays d'accueil qui reçoivent le plus grand nombre de personnes en quête d'une protection dans le Monde. Il existe un fort déséquilibre démographique entre des États tels l'Iran, le Soudan, la République Démographique du Congo... et des pays développés où les requérants sont relativement peu nombreux (Danemark, France, Grande Bretagne...). Par ailleurs, les réfugiés théoriquement protégés par la convention de Genève de 1951, sont en pratique de plus en plus considérés par les États européens, comme des migrants illégaux et traités comme tels. Au cours de ces dernières années, l'image du réfugié fait en effet de plus en plus l'objet d'un usage intempestif dans le langage commun, dès lors que ces exilés franchissent les frontières de l'Union européenne. L'image du réfugié reste toutefois revalorisée lorsqu'ils restent dans des États voisins de leur pays d'origine ou dans des régions «sûres" de leur État. Alors « le "droit de rester en sécurité dans son propre 
pays", selon la phraséologie consacrée, est aujourd'hui reconnu comme un droit fondamental que les démocraties doivent défendre (comme hier le droit de partir). C'est là le nouvel et véritable intérêt stratégique de la protection des victimes de persécution » (Legoux L., 2004, p. 19).

\section{BIBLIOGRAPHIE}

Agier Michel, 2002. Aux abords du monde, les réfugiés, Paris, Payot \& Rivages, 155 p.

Anafé, 2004. La frontière et le droit d'asile : la zone d'attente de Roissy sous le regard de l'Anafé bilan de six mois d'observation associative (avril-octobre 2004), Paris, 68 p. [disponible sur Internet]

http://www.Anafe.org/download/rapports/Anaf\%E9\%20Bilan\%20nov\%2004.pdf

Caloz-Tschopp Marie-Claire, ed., 1998. Hannah Arendt, les sans-Etats et le "droit d'avoir des droits », vol. 1, Paris-Montréal, L'Harmattan, 271 p.

Caloz-Tschopp Marie Claire, Axel Clevenot et Tschopp Maria-Pia, 1994. Asile, violence, exclusion en Europe : histoire, analyse, prospective, Genève, Cahiers de la Section des Sciences de l'Education de l'Université de Genève, Groupe de Genève « Violence et droit d'asile en Europe », 463 p.

Cambrezy Luc, 2001. Réfugiés et exilés : crise des sociétés, crise des territoires, Paris, IRD, 213 p.

Cambrezy Luc, Véronique Lassailly-Jacob, eds, 2001. Populations réfugiées, de l'asile au retour, Paris, IRD, $418 \mathrm{p}$.

Chemillé-Gendreau Monique, 1981. Le concept de réfugié en droit international, Pluriel, débat, vol. 28, p. 3-11.

Clochard Olivier, Mohammed Kamel Doraï, 2005. Aux frontières de l'asile : les réfugiés non palestiniens au Liban, a contrario, vol. 3, $\mathrm{n}^{\circ} 2$, p. 45-65.

Collovald Annie, ed, 2005. Etrangers : la mise à l'écart, Politix, nº 69, p.3-128.

$\mathrm{HCR}, 2000$, Les réfugiés dans le monde : cinquante ans d'action humanitaire, Paris, Autrement, $337 \mathrm{p}$.

Julien-Laferrière François, 2002. Le droit d'asile en question. Paris, La Documentation Française, $84 \mathrm{p}$.

Laacher Smaïn, 2007. Le peuple des clandestins, Paris, Calmann Lévy.

Lassailly-Jacob Véronique, Jean-Yves Marchal et André Quesnel, eds, 1999 Déplacés et réfugiés : la mobilité sous contrainte, Paris, IRD Editions, $504 \mathrm{p}$.

Legoux Luc, 2002. Vers une redéfinition du droit d'asile, Les cahiers français, $n^{\circ} 307$, p.77-84.

Legoux Luc, 2004. Changements et permanences dans la protection des réfugiés, Revue Européenne des Migrations Internationales, vol. 20, $\mathrm{n}^{\circ}$ 2, p.9-22.

Legoux Luc, 2006. Asile, Immigration : réconcilier les droits de l'homme et ceux du citoyen, Revue Européenne des Migrations Internationales, vol. 22, n² 2, p. 95-102. 
Le Pors Anicet, 2005. Le droit d'asile, Paris, PUF, 128 p.

Rodier Claire, 2006. Analyse de la dimension externe des politiques d'asile et d'immigration de l'UE-synthèse et recommandations pour le Parlement européen, [disponible sur Internet] http://www.europarl.europa.eu/meetdocs/2004_2009/documents/dt/619/619330/619330fr.pdf Rodier Claire, 2005. L'Europe et l'externalisation ; La Libye en première ligne, In Carobella exnatura, Politiques migratoires : grandes et petites manoeuvres, Marseille, p 47-60.

Simon Gildas, 1995. Géodynamique des migrations internationales dans le monde, Paris, Presses Universitaires de France, $429 \mathrm{p}$.

\section{NOTES}

1. 77 millions de personnes en 1965, 105 millions en 1985, 111 millions en 1990, 140 millions en 1997, 175 millions en 2000 et 191 millions en 2005.

2. 2,5 milliards de personnes sur la Terre dans les années $30 ; 4,1$ milliards en 1979; 5 milliards en 1987 et 6,5 milliards en 2006.

3. Anafé (Association Nationale d'Assistance aux Frontières pour les Etrangers) http:// www.Anafe.org/index.php

4. CFDA (Coordination Française pour le Droit d'Asile) http://cfda.rezo.net/

5. La Cimade (Service oecuménique d'entraide) http://www.cimade.org/

6. GISTI (Groupe d'Information et de Soutien des Immigrés) http://www.gisti.org/

7. 40620 demandes d'asile en 2004 contre 91200 en 1999 pour la Grande-Bretagne ; et 35610 demandes d'asile en 2004 contre 91110 en 1999 (source : Haut commissariat aux Réfugiés).

8. Discours du ministre de l'Intérieur français à l'ensemble des préfets, le 24 juillet 2006. Le texte peut être consulté à l'adresse suivante : http://www.interieur.gouv.fr/ misill/sections/a_l_interieur/le_ministre/interventions/reunion-prefets-24-07-06/ view

9. IRINnews, article du $1^{\mathrm{er}}$ juin 2006 « Report warns of deteriorating circumstances among Iraqi refugees » http://www.irinnews.org/report.asp?

ReportID=53644\&SelectRegion=Middle_East\&SelectCountry=SYRIA

10. L'Orient Le Jour du 19 avril 2007 « L'Irak s'engage à ne pas abandonner ses réfugiés l'UE donne de l'argent ".

11. Anthropologue à l'Ecole des hautes études et en sciences sociales.

12. Communiqué de l'Anafé du 22 août 2006 « Accueil des réfugiés libanais : larmes de crocodile et tentatives de refoulement en catimini "

13. Cf. dépêche AFP publiée dans le Monde du 19 avril 2006.

14. Lors de la Conférence des ministres de l'intérieur de la Méditerranée occidentale, qui s'est tenue à Nice les 11 et 12 mai 2006, sous la présidence de Nicolas Sarkozy, un communiqué des représentants souligne « les efforts des pays de la rive sud de la Méditerranée pour contenir l'émigration illégale vers l'Europe » (c'est nous qui soulignons). 15. Déclaration Universelle des Droits de l'Homme

16. Le texte " « Emigration illégale » : une notion à bannir » paru dans Libération du 13 juin 2006 sous la signature de Claire Rodier, présidente du réseau Migreurop, est à l'adresse http://www.migreurop.org/article922.html.

17. En 2006, la situation de sept demandeurs d'asile somaliens a fortement retenu l'attention de l'actualité lettone. Le statut de réfugié leur ayant été refusé en première 
instance, les sept Somaliens ont été maintenus dans le centre de rétention administrative d'Olaine (une ancienne prison) pendant une année. Après avoir fait appel de la décision des autorités lettone, le statut d'apatride leur a été finalement accordé. Selon le directeur du centre d'Olaine, cette situation pourrait conduire d'autres étrangers voire des terroristes, à revendiquer qu'ils proviennent de cette région, afin d'obtenir un titre de séjour en Lettonie (pays qui se caractérise par l'accueil d'un très faible nombre de demandeurs d'asile : 5 en 2003, 7 en 2004 et 20 en 2005). 18. Il suffit simplement d'être attentif aux travaux de l'Union européenne dans ce domaine. Un conseil des ministres s'est réuni début août 2004 pour rendre plus concret un programme pluriannuel sur l'immigration en Europe, le terrorisme et une politique commune de définition du réfugié et des critères d'octroi de l'asile.

\section{RÉSUMÉS}

Les distributions spatiales des personnes en quête d'une protection dans le Monde soulignent que les pays développés reçoivent un faible nombre de demandeurs d'asile et de réfugiés. Il existe un fort déséquilibre démographique entre des États tels aujourd'hui la Syrie, le Tchad ou le Soudan et des pays développés où les requérants sont relativement peu nombreux. Par ailleurs, les réfugiés théoriquement protégés par la convention de Genève de 1951, sont de plus en plus considérés par les États européens, comme des migrants illégaux et traités comme tels.

Spatial distributions of interns' displacement people (IDP) and refugees in the World underline that the developed countries receive a low number of this people. There is a strong demographic imbalance between States such today Syria, Chad or Sudan and countries developed where the applicants are relatively very few. In addition, the refugees theoretically protected by the convention of Geneva from 1951, are considered more and more by the European States, like migrants illegal and treated like such.

\section{INDEX}

Mots-clés : demandeurs d'asile, réfugiés, Union européenne

Keywords : Asylum applicant, refugees, European Union

\section{AUTEUR}

\section{OLIVIER CLOCHARD}

Olivier Clochard est Docteur en géographie. Il a soutenu sa thèse en 2007 sous la direction d'Emmanuel Ma Mung. L'intitulé en est «Le jeu des frontières dans l'accès au statut de réfugié: une géographie des politiques européennes d'asile et d'immigration ». Il est membre du Conseil d'Administration de Migreurop et participe au projet d'étude pour le Parlement européen sous la direction de l'agence STEPS Consulting Social et la CIMADE. Il a publié, entre autres, "The maritime border of Europe: upstream migratory controls" 
in Brunet-Jailly Emmanuel (2007) Borderlands, Ottawa, University of Ottawa Press, 2007, p.19-40 ;"Le jeu permanent des frontières dans le processus de mise à l'écart des "réfugiés" dans Bouquet Christian et Velasco-Graciet Hélène (2007) Regards géopolitiques sur les frontières, Paris, L'Harmattan (collection Géographie et Culture) 2007, p. 79-96 ; et, avec Kamel Doraï, Aux frontières de l'asile, les réfugiés non-palestiniens au Liban dans A Contrario, 2005, vol. 3, $\mathrm{n}^{\circ} 2$, p. 45-65). 